\title{
Optimizing current standard of care therapy for stage III non-small cell lung cancer
}

\author{
Vivek Verma, Steven H. Lin \\ Department of Radiation Oncology, University of Texas M.D. Anderson Cancer Center, Houston, TX, USA \\ Contributions: (I) Conception and design: SH Lin; (II) Administrative support: SH Lin; (III) Provision of study materials or patients: None; (IV) \\ Collection and assembly of data: All authors; (V) Data analysis and interpretation: All authors; (VI) Manuscript writing: All authors; (VII) Final \\ approval of manuscript: All authors. \\ Correspondence to: Steven H. Lin, MD, PhD. Department of Radiation Oncology, The University of Texas M.D. Anderson Cancer Center, 1515 \\ Holcombe Blvd, Unit 097, Houston, TX 77030, USA. Email: shlin@mdanderson.org.
}

\begin{abstract}
The management of stage III non-small cell lung cancer (NSCLC) remains complex and controversial, with a myriad of potentially feasible options. Given the diversity of non-surgical as well as surgical options, along with recent randomized data regarding adjuvant immunotherapy that has re-defined the standard of care for unresected stage III NSCLC cases, the goal of this narrative review was to provide a contemporary view at how management of these patients can be further optimized. Topics discussed include the following items: optimizing toxicity mitigation strategies (in order to avoid impaired receipt of subsequent therapies), the importance of multidisciplinary tumor boards (MTBs) and multidisciplinary clinics (MDCs), adhering to treatment approaches endorsed by national guidelines, prudently selecting patients for surgical intervention (as compared to non-operative approaches), coordination of multidisciplinary care so as to best preserve all potential therapeutic options, and addressing challenges regarding disparities in access to oncologic care. This review places particular emphasis for community and/or rural centers, which may not have the same level of resources and/or personnel as larger academic institutions. Taken together, these strategies are aimed towards the overarching goal of streamlining oncologic care for stage III NSCLC cases in light of the numerous approaches that currently exist for these patients.
\end{abstract}

Keywords: Access to care; durvalumab; immunotherapy; health disparities; non-small cell lung cancer (NSCLC)

Submitted Apr 26, 2020. Accepted for publication Jul 31, 2020.

doi: $10.21037 /$ tlcr-20-603

View this article at: http://dx.doi.org/10.21037/tlcr-20-603

\section{Introduction}

Although non-small cell lung cancer (NSCLC) most commonly presents as metastatic disease $(38 \%$ of new diagnoses), nonmetastatic cases most often occur as locally advanced stage III disease (28\% of new diagnoses) (1). Management of stage III NSCLC remains without clear consensus to date (2). These may be broadly divided based on surgical-based versus nonoperative approaches; the former has largely comprised of neoadjuvant chemotherapy or chemoradiotherapy (CRT) followed by resection, and the latter now refers to administering full-dose CRT followed by immunotherapy.
The evidence behind these diverse treatment paradigms for stage III cases stems from a lack of established level I evidence of overall survival (OS) benefits from several interventions. For instance, numerous randomized trials have failed to demonstrate higher OS when performing resection-based approaches as compared to full-dose CRT alone (3). Additionally, two randomized trials in the setting of resection have not discerned OS advantages to induction CRT versus chemotherapy alone $(4,5)$.

In the unresected setting, the publication of the randomized PACIFIC study has now demonstrated an OS improvement with addition of the anti-programmed death ligand-1 (PD-L1) antibody durvalumab (6,7). The 
PACIFIC trial randomized 713 patients with unresected stage III NSCLC to full-dose concurrent CRT followed by up to 12 months of durvalumab versus placebo. Key eligibility criteria included the lack of progression after CRT as well as an Eastern Cooperative Oncology Group (ECOG) performance status of $0-1$; of note, there were no such criteria related to PD-L1 level, assessment of which was not mandated. The median progression-free survival (PFS) was tripled at the time of last follow-up (17.2 vs. 5.6 months), and the 3 -year OS was $57 \%$ vs. $43.5 \%(6,7)$. Taken together, this remains one of the few instances of level I evidence supporting a particular intervention in locally advanced NSCLC.

Given that there are many potential options regarding management of stage III NSCLC, along with recent data that has re-defined the standard of care for some stage III NSCLC cases, the goal of this review was to provide a contemporary view at how management of these patients can be further optimized based on a comprehensive review of the existing literature in accordance with the Narrative Review Reporting Checklist (available at http://dx.doi. org/10.21037/tlcr-20-603).

\section{Optimizing delivery of the current standard of care}

In efforts to avoid delivering suboptimal therapy, it is first important to consider management approaches that have been proven suboptimal for stage III NSCLC based on previous research. This is especially important because initial therapies for NSCLC often highly influence subsequent management options.

Induction chemotherapy is sometimes delivered for high-risk cases, but it should be recognized that (based on randomized evidence) it does not improve outcomes and increases toxicities for nonoperatively managed locally advanced NSCLC (8). Because toxicities are often a deterrent to administering subsequent therapies in NSCLC, minimization of unnecessary toxicities during up-front therapy is essential.

Additionally, for non-operated cases, it should be recognized that decades of study have shown that concurrent CRT is the standard of care that best address locoregional disease $(9,10)$. Although single-modality therapy or sequential CRT is sometimes delivered to better spare patients from toxicities, implementing any of several toxicity management options (discussed in the next section) can maintain the ability to administer the current standard of concurrent CRT. The importance of being able to deliver concurrent therapy is of critical importance, as the PACIFIC investigation mandated concurrent CRT prior to immunotherapy and is reflective of the FDA approved indication. Thus, patients who undergo singlemodality therapy or sequential CRT may not only receive suboptimal therapy, but also may not be eligible to receive consolidation durvalumab after CRT.

For neoadjuvant cases planned for surgery, it is well recognized that surgery may not eventually occur, owing to a multitude of reasons, such as patient preference, toxicities, and tumor progression. If surgery is not pursued (an estimated $15-20 \%$ of cases), then transitioning to full-dose CRT and durvalumab would be the best option, provided that tumor progression has not occurred. Limiting delays during this transition is recommended in order to avoid tumor repopulation.

Lastly, some clinicians are utilizing up-front chemoimmunotherapy or immunotherapy alone in the neoadjuvant and/or nonoperative settings. While worthy of academic investigation, this is not the recognized standard of care at the present time. It is unclear whether these approaches would substitute for dedicated local control modalities such as radiotherapy. Although randomized trials are ongoing, experimental strategies such as these should not done outside the context of a clinical trial.

\section{Optimizing toxicity management during CRT}

As mentioned above, toxicities can sometimes impair receipt of subsequent therapies in stage III NSCLC; therefore, strategies for toxicity mitigation merit discussion in order to optimize therapy for these patients.

Contemporary radiation techniques are encouraged in efforts to reduce toxicities such as pneumonitis. A secondary analysis of the randomized RTOG 0617 trial showed a lower rate of grade $3+$ pneumonitis with the intensity modulated radiotherapy technique (11). The role of proton beam therapy for this purpose is unclear until RTOG 1308 is published (12-15), but can be considered based on encouraging phase II data in locally advanced NSCLC (16) along with prospective trials in other thoracic cancers $(17,18)$.

For other potential toxicities, prophylactically delivering supportive medications may also be beneficial (19). For instance, patients with disease abutting longer segments of the esophagus are at high risk of radiation esophagitis; which is critical to avoid, as it may lead to dehydration 
and weight loss. These patients may be managed with prophylactic proton pump inhibitors. Prophylactic drugs may avoid radiation treatment delays, which is a factor associated with poorer outcomes in NSCLC (20).

In addition to radiation treatment delays, experiencing CRT-related adverse events may result in delays in initiation, or lead to early discontinuation, of immunotherapy. In the PACIFIC trial, a post-hoc analysis revealed that patients had greater benefit of durvalumab if commenced 14 days from CRT completion. Although this should be taken with caution given the post hoc nature of the analysis, it is generally recommended that long (e.g., greater than 42 days) delays from CRT to immunotherapy are suboptimal. During the post-CRT interval, it is essential to promptly (e.g., ideally $\leq 14$ days) confirm the lack of progression with computed tomography imaging, as a prerequisite for PACIFIC was the lack of progression following CRT (if progression occurs, molecular testing may be required, as driver mutation-positive cases often do not respond well to immunotherapy). Positron emission tomography is not recommended in this setting owing to the high likelihood of residual avidity from recent CRT, thereby leading to a potentially higher rate of false-positives with regard to locoregional progression.

Lastly, management of immunotherapy related adverse events is essential to ensuring that the immunotherapy course is adequate for oncologic benefit. A number of expert panels have constructed detailed guidelines for this very purpose (21-23). Depending on the severity of the event, options include corticosteroids and/or suspending immunotherapy until symptoms improve, or permanent discontinuation of immunotherapy.

\section{Optimizing management through multidisciplinary care}

As therapy for locally advanced NSCLC most commonly involves bi- or tri-modality management, the importance of careful and seamless communication between multidisciplinary providers cannot be understated. Whereas patients treated at the vast majority of academic centers have more direct and streamlined access to surgical, radiation, and medical oncologists, this is not necessarily the case at some community and/or rural facilities, where referral patterns and wait times may lead to under-diagnosis, misdiagnosis, and/or delays from diagnosis to therapy. These can all profoundly impact outcomes of stage III NSCLC; the eventual administration of standard-of-care therapy may not adequately compensate for errors and/or delays in diagnostic processes. Additionally, multidisciplinary communication is essential to properly coordinating care that impacts downstream therapies. For instance, in the unresected setting, implementation of durvalumab based on approved indications is contingent on receiving concurrent CRT.

One intervention that may ameliorate the aforementioned challenges is the institution of a dedicated multidisciplinary tumor board (MTB). This often facilitates direct and clear communication between multidisciplinary providers, and often allows for a treatment plan to be formulated in a quicker manner, thereby potentially reducing delays from diagnosis to treatment. A retrospective analysis conducted by Freeman and colleagues of a 70-hospital database, comprising over 13,000 cases of stage I-III NSCLC, showed several corroboratory findings (24). Patients who had been evaluated at MTBs were more likely to have complete staging workup, receive therapy consistent with national guidelines, and experience less lag time between diagnosis and treatment. Treatment costs were also reduced in this group of patients. Taken together, these findings illustrate that MTBs improve the quality of care for these patients, can be particularly helpful in patients with "grey area" cases that may not fit a textbook definition of stage III NSCLC, and better facilitate optimal patient selection for a particular form of therapy (discussed below). Although many facilities may not have access to dedicated thoracic cancer MTBs, the utility of virtual MTBs has been rapidly emerging recently, especially with practice and policy adjustments made due to the COVID-19 pandemic (25). These have been shown for several disease sites to be feasible and effective for institutions without inhouse options (26-29).

In addition to MTBs, the institution of multidisciplinary clinics (MDCs) may provide additional advantages to patients who are newly diagnosed with locally advanced NSCLC. For patients, MDCs offer the advantage of meeting all providers up-front, which allows the entire treatment plan to be laid out as an aggregate at one time. This may have a profound psychological influence on patients, as up-front explanation of the treatment plan, as well as expectations thereof, may lead to a smoother transition between modalities of therapy and avoid treatment delays during that time period. Importantly, MDCs may also allow for a more balanced and equivocal discussion of management options with the patient and his/her family. Because treatment of stage III NSCLC is 
heterogeneous, MDCs may better facilitate the patients' understanding of the risks and benefits of a particular paradigm, and allowing them to make a choice of which regimen they prefer. Accounting for personal preferences of each individual patient in the care plan is infrequently considered, and more often it is as a result of physician bias towards a particular treatment paradigm. Although there is very little data or experience on conducting virtual MDCs in facilities without such in-house options, this should also be considered if feasible, provided that legal or technical restrictions are accounted for. The expansion of telemedicine, especially during the COVID-19 pandemic, may make this objective relatively easier, as providers from various locations could be able to provide efficient multidisciplinary evaluation of each patient.

\section{Opitimizing patient selection}

Multidisciplinary teams, ideally together with patient preference, are also important to select which patients are most optimal for a particular treatment paradigm. The most important example of this notion is careful evaluation for surgical eligibility in stage III NSCLC, since performing surgery eliminates the indication to deliver the level I evidence-supported durvalumab. Although some patients may benefit from elimination of locoregional disease burden by means of resection, there is currently no uniformly accepted approach on which patients would benefit most from surgery. As a result, it is critical to evaluate factors such as primary disease burden, mediastinal nodal disease burden, and patient/organ functional status for such interventions (30). Performing this evaluation in a multidisciplinary fashion, together with patient preferences after having a balanced discussion regarding the risks and benefits of surgical approaches, is highly important to adequately select patients for individualized approaches amidst numerous options thereof.

Of note, the PACIFIC trial protocol did not specifically define "resectability", as this is an extremely heterogeneous term that is dependent on individual countries, institutions, clinicians, and patient preferences. Hence, the term "unresectable" from that study implies the presence of numerous reasons for the lack of resection, but the application of the trial most relates to patients that are "unresected" for these various reasons.

Patient selection may also be exercised when considering the definition of stage III disease (and thus, eligibility for the aforementioned management options). For instance, assessment of nodal disease [e.g., with procedures such as endobronchial ultrasound (EBUS) or mediastinoscopy] impacts whether potential N2 disease is discovered prior to any interventions or at the time of surgical resection. Detecting occult N2 involvement prior to therapy preserves all therapeutic options (e.g., nonoperative versus surgicalbased therapy), whereas the same cannot be stated if N2 disease is incidentally discovered at the time of resection. Moreover, obtaining information about the degree of N2 station involvement can also impact management, as more extensive $\mathrm{N} 2$ disease may not benefit from resection owing to the high rate of distant metastatic potential (2). Furthermore, pathologic nodal assessment can also upstage suspected N2 disease to N3 disease, thereby avoiding futile surgical resection. A retrospective study from a single hospital network showed that pathologic nodal staging was more likely to be performed in patients seen at an MDC, and performing these procedures reduced the risk of detecting unsuspected N2 disease at the time of surgery over fourfold (31).

Additionally, patient selection is critical to evaluate whether unresected patients would be eligible for the PACIFIC trial. Because the trial did not include patients with an ECOG performance status of $\geq 2$, it is uncertain whether durvalumab would benefit these patients, as this cannot be extrapolated from the PACIFIC study. Additionally, assessment of progression after CRT is essential, as cases which progress likely have unfavorable biology and may not benefit from durvalumab.

\section{Opitimizing access to care}

It has been well-recognized that lung cancer patients with challenges in accessing oncologic care are more likely to receive therapy discordant with national guidelines, which is associated with poorer outcomes (32-34). Addressing this issue is crucial to improving outcomes for not only stage III NSCLC, but a myriad of other neoplasms as well.

Lacking access to surgeons and pulmonologists can hamper accurate diagnosis and disease assessment, especially given the importance of performing pathologic mediastinal nodal examination as mentioned above. This can lead to under-diagnosis and potential ineligibility for certain therapeutic interventions. Furthermore, these providers are often the first to see patients, and set up subsequent referrals; as a result, lack of access can substantially alter referral patterns and increase the lag time from diagnosis to therapy, which can negatively impact outcomes. 
Lack of access to radiation oncologists is also a concern, as radiotherapy is an essential component of nonoperative management. Lack of access to a radiation oncologist can result in guideline-discordant care such as the replacement of radiotherapy by off-label uses of immunotherapy in the neoadjuvant or nonoperative setting, which are not supported by randomized evidence and should be avoided. Delivering chemotherapy alone in the nonoperative setting, in the absence of a radiation oncologist, is not standard of care and reduces the indications/eligibility for durvalumab.

Lastly, as medical oncologists are responsible for delivering chemotherapy concurrent with radiotherapy as well as durvalumab in the unresected setting, lack of access to medical oncologists limits the ability to deliver a level I evidence-approved therapy. Many medical oncologists who may not be aware of post-CRT immunotherapy for unresected cases may still be administering post-CRT consolidation chemotherapy, which has no proven utility based on numerous prospective trials (35), and is no longer recommended in patients being treated with durvalumab (2). As a result, education of medical oncologists who may not be familiar with the PACIFIC data is also essential in efforts to avoid delivering non-standard therapy and compromising patient outcomes.

Improving access to oncologic providers may be better addressed by aforementioned notions regarding virtual MTBs or MDCs. Additionally, other disease sites have experienced success with mobile cancer screening, largely in rural areas (36). This most commonly consists of a vehicle, equipped with appropriate imaging capabilities for screening, travels to various rural communities in order to screen at-risk patients. Although lung cancer screening is relatively new as compared with screening for other disease sites, improved efforts are necessary to ensure that NSCLC is diagnosed as nonmetastatic disease, which is eligible for curative-intent therapy.

Taken together, although access to oncologic care in patients with lung cancer has been a well-known issue, educational initiatives for both providers and patients are highly recommended in efforts to ensure that the quality of therapy in underserved areas is consistent with nationally established practices. These can be utilized together with the principles of multidisciplinary management as mentioned above.

\section{Conclusions}

The management of stage III NSCLC remains complex with a myriad of options. Given the diversity of surgical and non-surgical options, along with recent randomized data that has re-defined the standard of care for unresected stage III NSCLC cases, the goal of this review was to provide a contemporary view at how management of these patients can be further optimized. Topics discussed include optimizing toxicity mitigation strategies (to avoid impaired receipt of subsequent therapies), the importance of MTBs and MDCs, adhering to treatment approaches endorsed by national guidelines, adequately selecting patients for surgical intervention as compared to nonoperative approaches, coordination of multidisciplinary care so as to best preserve all potential therapeutic options, and addressing challenges regarding disparities in access to oncologic care.

\section{Acknowledgments}

The authors thank Ion Cotarla, Tiernan Mulrooney, and Doris Makari-Anders from AstraZeneca for review and editing the manuscript.

Funding: None.

\section{Footnote}

Reporting Checklist: The authors have completed the Narrative Review Reporting Checklist. Available at http:// dx.doi.org/10.21037/tlcr-20-603

Conflicts of Interest: Both authors have completed the ICMJE uniform disclosure form (available at http://dx.doi. org/10.21037/tlcr-20-603). Dr. SHL serves as an unpaid editorial board member of Translational Lung Cancer Research from Sep 2019 to Sep 2021. Representatives from AstraZeneca contributed edits to this manuscript. SHL has received research funding from Elekta, STCube Pharmaceuticals, Peregrine, Hitachi Chemical Inc., and Roche/Genentech, has served on the advisory board for AstraZeneca, and received honoraria from US Oncology and ProCure. VV has no conflicts of interest to declare.

Ethical Statement: The authors are accountable for all aspects of the work in ensuring that questions related to the accuracy or integrity of any part of the work are appropriately investigated and resolved.

Open Access Statement: This is an Open Access article distributed in accordance with the Creative Commons Attribution-NonCommercial-NoDerivs 4.0 International 
License (CC BY-NC-ND 4.0), which permits the noncommercial replication and distribution of the article with the strict proviso that no changes or edits are made and the original work is properly cited (including links to both the formal publication through the relevant DOI and the license). See: https://creativecommons.org/licenses/by-nc-nd/4.0/.

\section{References}

1. Morgensztern D, Ng SH, Gao F, et al. Trends in Stage Distribution for Patients with Non-Small Cell Lung Cancer: A National Cancer Database Survey. J Thorac Oncol 2010;5:29-33.

2. National Comprehensive Cancer Network. Non-Small Cell Lung Cancer. Version 6.2020. Available online: https://www.nccn.org/professionals/physician_gls/pdf/ nscl.pdf. Accessed July 23, 2020.

3. Pöttgen C, Eberhardt W, Stamatis G, et al. Definitive radiochemotherapy versus surgery within multimodality treatment in stage III non-small cell lung cancer (NSCLC) - a cumulative meta-analysis of the randomized evidence. Oncotarget 2017;8:41670-8.

4. Thomas M, Rube C, Hoffknecht P, et al. Effect of preoperative chemoradiation in addition to preoperative chemotherapy: a randomised trial in stage III non-smallcell lung cancer. Lancet Oncol 2008;9:636-48.

5. Pless M, Stupp R, Ris HB, et al. Induction chemoradiation in stage IIIA/N2 non-small-cell lung cancer: a phase 3 randomised trial. Lancet 2015;386:1049-56.

6. Antonia SJ, Villegas A, Daniel D, et al. Overall Survival with Durvalumab after Chemoradiotherapy in Stage III NSCLC. N Engl J Med 2018;379:2342-50.

7. Antonia SJ, Villegas A, Daniel D, et al. Durvalumab after Chemoradiotherapy in Stage III Non-Small-Cell Lung Cancer. N Engl J Med 2017;377:1919-29.

8. Vokes EE, Herndon JE, Kelley MJ, et al. Induction Chemotherapy Followed by Chemoradiotherapy Compared With Chemoradiotherapy Alone for Regionally Advanced Unresectable Stage III Non-small-cell Lung Cancer: Cancer and Leukemia Group B. J Clin Oncol 2007;25:1698-704.

9. Curran WJ Jr, Paulus R, Langer CJ, et al. Sequential vs. Concurrent Chemoradiation for Stage III Non-Small Cell Lung Cancer: Randomized Phase III Trial RTOG 9410. J Natl Cancer Inst 2011;103:1452-60.

10. Bradley JD, Hu C, Komaki RR, et al. Long-Term Results of NRG Oncology RTOG 0617: Standard- Versus HighDose Chemoradiotherapy With or Without Cetuximab for Unresectable Stage III Non-Small-Cell Lung Cancer. J Clin Oncol 2020;38:706-14.

11. Chun SG, Hu C, Choy H, et al. Impact of IntensityModulated Radiation Therapy Technique for Locally Advanced Non-Small-Cell Lung Cancer: A Secondary Analysis of the NRG Oncology RTOG 0617 Randomized Clinical Trial. J Clin Oncol 2017;35:56-62.

12. Verma V, Chang JY. Controversies in Dose-Escalation for Locally Advanced Non-Small Cell Lung Cancer and the Role of Proton Beam Therapy. J Thorac Dis 2018;10:S1124-S1126.

13. Cushman TR, Verma V, Rwigema JCM. Comparison of Proton Therapy and Intensity Modulated Photon Radiotherapy for Locally Advanced Non-Small Cell Lung Cancer: Considerations for Optimal Trial Design. J Thorac Dis 2018;10:S988-S990.

14. Verma V, Chang JY. Dose-escalation of Locally Advanced Non-Small Cell Lung Cancer With Proton Beam Therapy. Transl Lung Cancer Res 2018;7:S280-S282.

15. Brooks ED, Ning MS, Verma V, et al. Proton Therapy for Non-Small Cell Lung Cancer: The Road Ahead. Transl Lung Cancer Res 2019;8:S202-S212.

16. Chang JY, Verma V, Li M, et al. Proton Beam Radiotherapy and Concurrent Chemotherapy for Unresectable Stage III Non-Small Cell Lung Cancer: Final Results of a Phase 2 Study. JAMA Oncol 2017;3:e172032.

17. Chen D, Menon H, Verma V, et al. Results of a Phase 1/2 Trial of Chemoradiotherapy With Simultaneous Integrated Boost of Radiotherapy Dose in Unresectable Locally Advanced Esophageal Cancer. JAMA Oncol 2019;5:1597-604.

18. Lin SH, Hobbs BP, Verma V, et al. Randomized Phase IIB Trial of Proton Beam Therapy Versus Intensity-Modulated Radiation Therapy for Locally Advanced Esophageal Cancer. J Clin Oncol 2020;38:1569-79.

19. Verma V, Simone CB 2nd, Werner-Wasik M. Acute and Late Toxicities of Concurrent Chemoradiotherapy for Locally-Advanced Non-Small Cell Lung Cancer. Cancers (Basel) 2017;9:120.

20. McMillan MT, Ojerholm E, Verma V, et al. Radiation Treatment Time and Overall Survival in Locally Advanced Non-small Cell Lung Cancer. Int J Radiat Oncol Biol Phys 2017;98:1142-52.

21. Puzanov I, Diab A, Abdallah K, et al. Managing Toxicities Associated With Immune Checkpoint Inhibitors: Consensus Recommendations From the Society for Immunotherapy of Cancer (SITC) Toxicity Management Working Group. J Immunother Cancer 2017;5:95. 
22. Brahmer JR, Lacchetti C, Schneider BJ, et al. Management of Immune-Related Adverse Events in Patients Treated With Immune Checkpoint Inhibitor Therapy: American Society of Clinical Oncology Clinical Practice Guideline. J Clin Oncol 2018;36:1714-68.

23. Thompson JA, Schneider BJ, Brahmer J, et al. NCCN Guidelines Insights: Management of ImmunotherapyRelated Toxicities, Version 1.2020. J Natl Compr Canc Netw 2020;18:230-41.

24. Freeman RK, Ascioti AJ, Dake M, et al. The Effects of a Multidisciplinary Care Conference on the Quality and Cost of Care for Lung Cancer Patients. Ann Thorac Surg 2015;100:1834-8.

25. Salari A, Shirkhoda M. COVID-19 pandemic \& head and neck cancer patients management: The role of virtual multidisciplinary team meetings. Oral Oncol 2020;105:104693.

26. Marshall CL, Petersen NJ, Naik AD, et al. Implementation of a Regional Virtual Tumor Board: A Prospective Study Evaluating Feasibility and Provider Acceptance. Telemed J E Health 2014;20:705-11.

27. Shea CM, Teal R, Haynes-Maslow L, et al. Assessing the Feasibility of a Virtual Tumor Board Program: A Case Study. J Healthc Manag 2014;59:177-93.

28. Salami AC, Barden GM, Castillo DL, et al. Establishment of a Regional Virtual Tumor Board Program to Improve the Process of Care for Patients With Hepatocellular Carcinoma. J Oncol Pract 2015;11:e66-e74.

29. Abu Arja MH, Stanek JR, Morales la Madrid AE, et al. The Latin American Brain Tumor Board Teleconference:
Results of a Web-Based Survey to Evaluate Participant Experience Utilizing This Resource. Childs Nerv Syst 2019;35:257-65.

30. Haque W, Verma V, Butler EB, et al. Post-treatment Mortality After Definitive Chemoradiotherapy Versus Trimodality Therapy for Locally Advanced Non-Small Cell Lung Cancer. Lung Cancer 2019;127:76-83.

31. Friedman EL, Kruklitis RJ, Patson BJ, et al. Effectiveness of a thoracic multidisciplinary clinic in the treatment of stage III non-small-cell lung cancer. J Multidiscip Healthc 2016;9:267-74.

32. Goulart BH, Reyes CM, Fedorenko CR, et al. Referral and treatment patterns among patients with stages III and IV non-small-cell lung cancer. J Oncol Pract 2013;9:42-50.

33. Lathan CS. Lung cancer care: the impact of facilities and area measures. Transl Lung Cancer Res 2015;4:385-91.

34. Ahmed HZ, Liu Y, O'Connell K, et al. Guidelineconcordant Care Improves Overall Survival for Locally Advanced Non-Small-cell Lung Carcinoma Patients: A National Cancer Database Analysis. Clin Lung Cancer 2017;18:706-18.

35. Tsujino K, Kurata T, Yamamoto S, et al. Is consolidation chemotherapy after concurrent chemo-radiotherapy beneficial for patients with locally advanced non-small-cell lung cancer? A pooled analysis of the literature. J Thorac Oncol 2013;8:1181-9.

36. Greenwald ZR, El-Zein M, Bouten S, et al. Mobile Screening Units for the Early Detection of Cancer: A Systematic Review. Cancer Epidemiol Biomarkers Prev 2017;26:1679-94.
Cite this article as: Verma V, Lin SH. Optimizing current standard of care therapy for stage III non-small cell lung cancer. Transl Lung Cancer Res 2020;9(5):2033-2039. doi: 10.21037/tlcr20-603 